Cite as:

Domènech-Abella, J., Mundó, J., Haro, J.M., Rubio-Valera, M., (2019) Anxiety, depression, loneliness and social network in the elderly: longitudinal associations from The Irish Longitudinal Study on Ageing (TILDA), Journal of Affective Disorders, 246: 82-88 doi: 10.1016/j.jad.2018.12.043

\title{
Anxiety, depression, loneliness and social network in the elderly: longitudinal associations from The Irish Longitudinal Study on Ageing (TILDA)
}

Joan Domènech-Abella ${ }^{123}$, Jordi Mundó ${ }^{2}$, Josep Maria Haro ${ }^{134}$, Maria Rubio-Valera ${ }^{156}$

1. Parc Sanitari Sant Joan de Déu, Sant Boi de Llobregat, Barcelona, Spain.

2. Department of Sociology, Universitat de Barcelona, Barcelona, Spain.

3. Instituto de Salud Carlos III, Centro de Investigación Biomédica en Red de Salud Mental (CIBERSAM), Madrid, Spain.

4. Instituto de Investigación Sanitaria Princesa (IP), Madrid, Spain.

5. Instituto de Salud Carlos III, Centro de Investigación Biomédica en Red de Epidemiología y Salud Pública (CIBERESP), Madrid, Spain.

6. Faculty of Pharmacy and Food Science, Universitat de Barcelona, Barcelona, Spain.

*Corresponding author:

Joan Domènech-Abella, MSc

Research, Innovation and Teaching Unit, Parc Sanitari, Sant Joan de Déu

Dr. Antoni Pujadas, 42, Sant Boi de Llobregat, Barcelona, Spain

E-mail: j.domenech@pssjd.org

Tel: (+34) 9355696 77; Fax: (+34) 936520051 
Anxiety, depression, loneliness and social network among middle-aged and older people: longitudinal associations from The Irish Longitudinal Study on Ageing (TILDA)

\begin{abstract}
Background: Social network, loneliness, generalized anxiety disorder (GAD) and major depression disorder (MDD) are interrelated. However, as the directions of these associations are still unclear, we examined them prospectively using community-based data.
\end{abstract}

Methods: Data on 5066 adults aged $\geq 50$ years from the Irish Longitudinal Study on Ageing (TILDA) were analyzed. Loneliness was assessed through the UCLA Loneliness Scale. Social integration was measured using the Berkman-Syme Social Network Index. MDD and GAD were assessed with the Composite International Diagnostic Interview (CIDI). Logistic regression models were conducted.

Results: The longitudinal association between experiencing loneliness and higher likelihood of suffering from MDD or GAD two years later is bidirectional but stronger with loneliness as origin, whereas the association between social isolation and higher likelihood of subsequent MDD or GAD as well as those between loneliness and subsequent deterioration of social integration are unidirectional.

Conclusion: Objective and perceived social isolation independently affect the probability of suffering from MDD or GAD whereas loneliness is a risk factor for the deterioration of social life, which highlights the need to address the subjective factors (such as loneliness) and objective factors (such as social network size) of social isolation in a complementary way in order to improve the mental health of the older adult population.

Keywords: depression; anxiety; social network; loneliness; Ireland. 


\section{Introduction}

Major depressive disorder (MDD) and generalized anxiety disorder (GAD) are among the most prevalent mental disorders among middle-aged and older people (hereinafter referred to as "older adults") (Beekman et al., 1998; Byers et al., 2010). In Ireland, the 12-month prevalence of MDD and GAD in adults ages 45 to 64 has been reported to be $7 \%$ and $4 \%$ respectively, while among those aged 65 years and over, it is $3 \%$ and $1 \%$, respectively (Barry et al., 2009). MDD has a significant impact on older populations and is linked to higher risk of suicide, all-cause mortality and increasing health service use (Blazer, 2003). Similarly, GAD is associated with chronic medical conditions, a higher burden on the health care system and lower health-related quality of life (Porensky et al., 2009).

According to a systematic review, the main predictors of depressive disorders and symptoms in the elderly are: female gender, somatic illness, cognitive impairment, functional disability, lack or loss of close social contacts and clinical history of depression (Djernes, 2006). Another comprehensive review that also assessed risk factors for anxiety revealed that few studies have explored this issue. The findings of these studies showed that risk factors for anxiety are similar to those for depression although biological factors seemed more important in predicting depression than anxiety. The study also showed that social factors affected depression and anxiety differently: low contact frequency and being childless were associated with anxiety, whereas smaller network size and being unmarried were associated with depression (Vink et al., 2008). Moreover, anxiety and depressive disorders have been identified as bidirectional risk factors for one another (Jacobson and Newman, 2017).

Poor mental health has clearly been linked to objective social isolation (Levula et al., 2016; Thoits, 2011), as well as to subjective measures such as perceived social 
support or loneliness, which have been of increasing interest to researchers over the last few decades decades (Wang et al., 2018). Social isolation is defined as a measurable lack of social relationships, while loneliness is synonymous with perceived social isolation, not with objective social isolation (Hawkley et al. 2010). Approximately 10\% of older adults suffer from "chronic loneliness" in Ireland and about 7\% reported being socially isolated in the baseline of the Irish Longitudinal Study on Ageing, of which 60\% said they do not feel lonely (Harvey \& Walsh, 2016).

In older adults, social isolation and loneliness have been shown to negatively affect a range of health outcomes, including all-cause mortality (Pantell et al., 2013; Rico-Uribe et al., 2016; Steptoe et al., 2013). Being female, previously married, unemployed, with a low educational level, low household income, and living in a rural area are sociodemographic factors associated with a higher likelihood of social isolation and loneliness among older adults (Cohen-Mansfield et al., 2016).

Although the association between social isolation and loneliness has been found to be only weak-to-moderate among older adults (Cornwell and Waite, 2009), most researchers presuppose that loneliness is caused by social isolation (Dahlberg et al., 2018; Routasalo et al., 2006). However, according to the Evolutionary Theory of Loneliness (ETL), the perception of being socially isolated (i.e., lonely) causes "behaviors categorized in terms of evolutionary fitness as selfish" (Cacioppo et al., 2018). In other words, the individual unconsciously attributes the feeling of loneliness to a negative perception of their social context that fosters selfish behavior. Therefore, the adaptive functions of loneliness that foster short-term survival, in the modern world can have long-term deleterious consequences and loneliness could cause an increase in social isolation. 
Although the association between social network and depression is widely recognized (Santini et al., 2015), whether loneliness causes depression or depression increases the feelings of loneliness, or both, has not been fully established and contrasting results have been reported (Cacioppo et al., 2010; Dahlberg et al., 2014; Domènech-Abella et al., 2017a). The relationship between anxiety and factors related to social network has been studied less than the relationship with depression but researchers have taken into account both directions of these associations. Anxiety disorders influence social support, contact with family of origin and neighborhood quality (Cramer et al., 2005). Social disability in patients with depression or anxiety predicts a diagnosis of depression or anxiety two years later (Saris et al., 2017).

The Irish Longitudinal Study on Ageing (TILDA) showed that loneliness was a significant mediator in the association between social network components and symptoms of depression but not anxiety symptoms (Santini et al., 2016). In contrast, in a longitudinal study conducted in Chicago, researchers reported that the temporal association between loneliness and depression was not attributable to the size or quality of social networks (Cacioppo et al., 2010) and, in the context of the Longitudinal Aging Study Amsterdam, loneliness and social network were found to have an independent effect on the course of depression (Houtjes et al., 2014).

Although most studies report anxiety as predictor of depression, a recent metaanalysis proposed that anxiety and depression symptoms are bi-directionally and prospectively associated with one another strongly over shorter time periods and weakly over longer time periods (Jacobson \& Newman, 2017). Relational factors such as mechanisms of these asssociations have been found taking GAD or anxiety symptoms as predictive of depression. For example, low perceived emotional social support have been found to be mediator in the association between depressive and anxiety symptoms 
48 months later for bereaved individuals (Jacobson, et al., 2017b), interpersonal oversensitivity and social chronic stress have been found as mediating the relationship between GAD before age 15 and later depression (Starr et al., 2014), and perceptions of not being accepted or loved were found to significantly mediate the relationship between adolescent anxiety and clinical depression in adulthood (Jacobson \& Newman, 2016). However, no prior research has examined the mechanism of this relationship in an older adult population.

Our aim was to test longitudinal associations, taken from the contrasting approaches in the literature reviewed, in which MDD, GAD, social networks and loneliness were involved: a) the association between MDD and GAD and changes in loneliness and vice versa, b) the association between affective disorders and changes in social network and vice versa, c) the association between social networks and changes in loneliness and vice versa. Following the literature reviewed on the causal mechanisms between GAD and MDD, we hypothesized that GAD would precede social isolation and feelings of loneliness and that these would lead to MDD, whereas the longitudinal association between GAD and MDD would be bidirectional. However, we were not able to provide a clear hypothesis regarding the relationship between social isolation and loneliness due to the contradictory approaches.

\section{Methods}

\section{Study Design}

The Irish Longitudinal Study on Ageing (TILDA) is a large prospective study of the social, economic and health circumstances of community-dwelling adults aged 50 years and older in Ireland. Details of sampling and study design have been described previously (Whelan and Savva, 2013). The first wave was collected between October 2009 and July 2011 with 8,175 eligible participants aged 50 and over and 329 younger 
partners. Participants completed a computer-assisted personal interview (CAPI) and were asked to fill in a self-completion questionnaire (SCQ) and return it by post. The overall household response rate to the CAPI at Wave 1 was $62 \%$ participants of which 85\% returned the SCQ. The second wave was collected between April 2012 and January 2013. An overall response rate of $86 \%$ was achieved and $84 \%$ of Wave 2 respondents returned the SCQ. The third wave was collected between March 2014 and October 2015. The overall response rate was $85 \%$ participants of which $85 \%$ returned the SCQ.

As baseline measurements of MDD and GAD were not taken, the present analysis is based on data obtained in wave 2 and wave $3(n=6,189)$. Among them, those younger than 50 years old $(n=275)$, with missing values on SNI components $(n=163)$ and those who did not complete the SCQ (including those with several cognitive impairment) ( $\mathrm{n}=685)$ were excluded. The last inclusion criterion is justified since SCQ included the UCLA loneliness scale (Russell, 1996). The sample size after restriction of these individuals was 5,066 (see Figure 1).

\section{Ethics statement}

Ethics approval was obtained from the Faculty of Health Sciences Research Ethics Committee at Trinity College, Dublin and participants provided written informed consent.

\section{Measures}

The Composite International Diagnostic Interview - Short Form (CIDI-SF) was used by lay interviewers for the assessment of MDD in the past 12 months and GAD lasting six months or longer. More information on the CIDI is available elsewhere (Kessler et al., 1998).

There is no consensus on how to measure social isolation / integration. However, we have used the Berkam-Syme Social Network Index (SNI) (Berkman and Syme, 
1979), which has been used by several researchers (Santini et al., 2016; Cacippo et al., 2010). Four dichotomous variables were summed to generate a total SNI: being married or living with a partner, having close friends or relatives, belonging to a voluntary group or club, attending church services at least twice a month. The composite score ranged from 0 to 4 and was classified according to the standard categorization: $0-1$ as most isolated, 2 as moderately isolated, 3 as moderately integrated, and 4 as most integrated.

There is no consensus on the dimensional nature of loneliness. For the present study, it is considered as a one-dimensional construct, as the researchers at the University of California-Los Angeles (UCLA) proposed (Russell, 1996). The UCLA loneliness scale is a 5-item scale including "How often do you feel isolated?" and “How often do you feel lonely?', Response categories ranged from 0 (hardly ever or never) to 2 (often). The scores were summed to create a scale ranging from 0 to 10 , with higher scores indicating greater levels of loneliness.

The internal validity of the loneliness scale was assessed using Cronbach's alpha. Correlation between loneliness scale and SNI was also evaluated. The scale showed good internal consistency $(\alpha=0.79)$ while the correlation was low $(r=0.20)$ indicating adequate discriminant validity between loneliness scale and SNI.

Sociodemographic characteristics included age, sex, education, financial circumstances, widowhood, employment status and place of residence. Age was categorized as $50-59,60-69,70-79$, and $\geq 80$ years old. Education was classified as: primary (some primary / not complete; primary or equivalent); secondary (intermediate / junior / group certificate or equivalent; leaving certificate or equivalent); and tertiary (diploma / certificate; primary degree; postgraduate / higher degree). Financial strain was assessed by responding to the statement "shortage of money stops me from doing the things I want to do" and answers were coded as follows: 1 (never); 2 (rarely); 3 
(sometimes); and 4 (often). Employment status was categorized as employed, retired / disabled, unemployed, homemaker and other. Place of residence was dichotomized into urban (Dublin city or county / another town or city) and rural.

According to the CAPI questionnaire, we distinguished heart diseases and remaining chronic, somatic conditions and two indicators were created: heart diseases and other diseases. In both cases, the number of chronic conditions was assessed through the question: "has a doctor ever told you that you have any of the conditions on this card?". In the case of heart diseases, responses included 11 conditions: High blood pressure or hypertension, angina, heart attack (including myocardial infarction or coronary thrombosis), congestive heart failure, diabetes or high blood sugar, stroke (cerebral vascular disease), ministroke or transient ischemic attack, high cholesterol, heart murmur, abnormal heart rhythm, and any other heart trouble. In the case of remaining somatic diseases, responses includes 9 conditions: chronic lung disease such as chronic bronchitis or emphysema, asthma, arthritis (including osteoarthritis, or rheumatism), osteoporosis, sometimes called thin or brittle bones, cancer or a malignant tumor (including leukemia or lymphoma but excluding minor skin cancers), serious memory impairment, stomach ulcers, varicose ulcers (an ulcer due to varicose veins), and cirrhosis, or serious liver damage. In both cases, the total number of chronic medical conditions was calculated and categorized as 0 (none), 1-2 and $\geq 3$.

\section{Statistical analysis}

The statistical analyses took into account the stratified study design. Sampling weights were generated, adjusting for the population distribution obtained from the national census and for non-response in order to obtain nationally-representative estimates (Moussavi et al., 2007). 
More than 12 percent of individuals did not complete the UCLA loneliness Scale. We cannot be certain about the reasons for the missing data, but no major discrepancy was found between imputed data and complete-case analysis so we are leaning towards imputed data as missing at random. Missing values were imputed using multiple imputation by chained equations through the predictive mean matching method. The imputation model included important sociodemographic and health-related variables associated with drop-outs. Thirty imputed databases were created. The number of imputations was calculated using a rule of thumb with respect to the fraction of missing information (M>100*FMI) (Rubin, 2004).

Descriptive analyses were conducted to characterize the study sample These analyses included weighted proportions and unweighted frequencies. The stability of outcome variables was analyzed by calculating the kappa coefficient between wave 2 and wave 3 and suggesting the following interpretations (Landis and Koch, 1977): 0.00 - 0.20 Slight; 0.21 - 0.40 Fair; $0.41-0.60$ Moderate; $0.61-0.80$ Substantial; and 0.81 - 1.00 Almost perfect. In addition, the proportion of individuals that improved, got worse, or remained stable were plotted for each outcome variable.

Logistic regression models were fitted to test the relationship between SNI or loneliness with each affective disorder, whereas ordered logistic regression models were used to assess differences in the likelihood of suffering from MDD or GAD and level of SNI or loneliness. In all cases, outcome variables were fitted at wave 3 and adjusted for the same variable at wave 2. Later, the models were also adjusted for all covariates (age cohorts, place of residence, employment status, financial strain, education, heart diseases, somatic diseases, affective disorders, loneliness, and SNI). Odds ratio (95\% confidence interval) and significance when $\mathrm{p}<0.05$ were reported in each model.

\section{Results}


The mean (SE) age at baseline was $63.3(0.16)$ years and $51.8 \%$ of the sample were women. Study sample characteristics are presented in Table 1. Table 2 shows outcome variable score stability between Wave 2 and Wave 3. SNI showed a lower percentage of participants improving from wave 2 to wave $3(9.7 \%$ among the isolated and $5.8 \%$ among the integrated) whereas loneliness showed a higher percentage $(23.7 \%$ among those with or without some loneliness and $64.4 \%$ among those with higher levels of loneliness). Among participants who suffered from MDD or GAD at wave 2, $22.8 \%$ and a $25.1 \%$, respectively, presented no active episode at wave 3 . SNI showed substantial agreement between two waves (kappa>0.61), whereas for remaining outcome variables the agreement was fair (kappa between 0.21 and 0.40 ).

Logistic regression models (Table 3 and Table 4) reported factors at wave 2 (as covariates) related to depression, anxiety, loneliness and SNI at wave 3 (as outcomes). In Table 3 each variable was adjusted for the outcome of each model at wave 2. A lower level of loneliness at wave 2 was associated with a higher probability of having depression, anxiety and a lower level of social isolation at wave 3. Suffering from affective disorders at wave 2 was associated with suffering from them at wave 3 and with worsening levels of loneliness and social integration. Finally, a higher level of social integration at wave 2 was related to a lower probability of suffering from depression or anxiety at wave 3 and, to a lesser extent, with worse levels of loneliness.

In Table 4, factors were also adjusted between one another and for the remaining covariates shown in Table 1. All the factors tested in Table 3 remained significantly associated with the outcomes, apart from SNI and depression in Model 3 (with loneliness as outcome) and both affective disorders in Model 4 (with SNI as outcome).

\section{Discussion}


The present study analyzed hypothetical bidirectional longitudinal associations involving affective disorders, social network index (SNI) or feeling of loneliness. The aim was to clarify the contrasting results found in the literature reviewed. Partially supporting our hypothesis, the longitudinal association between MDD and GAD was found to be bidirectional whereas GAD but not MDD predicted loneliness. However, neither GAD nor MDD predicted objective social isolation whereas loneliness and objective social isolation predicted MDD and GAD.

The results showing loneliness as a predictor of changes in social isolation but not vice versa is in contrast with several studies. Although social network related factors have normally been considered as predictors of loneliness (Dahlberg et al., 2018; Routasalo et al., 2006), some researchers report that loneliness and social isolation have only a weak-to-moderate positive correlation (Cornwell and Waite, 2009) among the oldest adults and suggest that it is due to a preparation of the individual for diminished social networks when these oldest adults begin experience impairment in the physical and mental capacities needed for social engagement (Achenbaum and Bengtson, 1994; Cornwell and Waite, 2009). Some researchers also observe that the prevalence of solitary living increases with age whereas feelings of loneliness decrease (Stepler, 2016). The absence of evidence indicating increasing loneliness and decreasing psychosocial well-being in the aging process are associated with the idea that older people can do as well or sometimes even better than young people with regard to happiness or self-acceptance (Domènech-Abella et al., 2017b, 2017c). In addition, according our results, the hypothetic decoupling between loneliness and social networks in the old age could be also due to an unidirectional association of high levels of loneliness with subsequent worsening social network characteristics, which could be explained using the Evolutionary Theory of Loneliness, according to which people tend 
to maintain selfish behavior when they feel loneliness, which harms their long-term social environment (Cacioppo et al., 2018): therefore, among the oldest adults, there could be increasing social isolation but not loneliness.

Apart from individual factors such as feelings of loneliness, social isolation is also induced by contextual factors such as limited opportunities to participate in social activities (Toepoel, 2013) and structural factors such as economic and social policies that produce and maintain socioeconomic inequalities (Nicholson, 2012). This could explain the high perpetuity of social isolation revealed by the results of the present study. In the field of interventions to reduce or prevent social isolation in later life, a recent review pointed out that social isolation reduction strategies such as one-to-one or group interventions seem to be less efficient in reducing social isolation than preventive strategies such as neighborhood or structural interventions (Cotterell et al., 2018).

The finding that low levels of SNI, and a higher level of loneliness in particular, are associated with a greater likelihood of subsequent generalized anxiety or major depression disorder is consistent with a recent case-control study on indicators of social functioning, using data from the Netherlands Study of Depression and Anxiety (NESDA). In this study, behavioral (such as components of SNI) and, with major intensity, affective indicators (such as loneliness) were found to be predictive of clinical anxiety or depression 2 years later, and especially in patients with comorbid disorders (Saris et al., 2017). In line with these results, in a systematic review Santini et al. (2015) highlighted the significant protective effects against depression of perceived support, and to a lesser degree, received support.

Anxiety and depression disorders are comorbid in a high proportion, which has been quantified as between $60 \%$ and $70 \%$ among depression or anxiety patients (Lamers et al., 2011). This high comorbidity can cause confusion about the effects on loneliness 
of each disorder. According to the present study, MDD is not significantly related to changes in loneliness after adjusting the association for GAD in the logistic regression model, whereas neither disorder was found to be significantly associated with changes in SNI.

According to the present study, and in line with researchers who suggested an independent effect of loneliness and social isolation on mental health (Cacioppo et al., 2010; Houtjes et al., 2014), objective and perceived social isolation independently affect the probability of suffering anxiety or depression whereas loneliness is a risk factor for the deterioration of social life, which highlights the need to address the subjective factors and objective factors of social isolation in a complementary way with the aim of improving the mental health of the older adult population.

\section{Limitation and strengths}

The strengths of this study include the use of a large nationally representative sample of older adults with a heterogeneous socioeconomic background, the inclusion of covariates, and the longitudinal design that enables us to examine time relationships. However, we need to consider several limitations. First, comparability across studies is difficult given the measurement inconsistencies among them. Second, information on components of SNI was missing in about $7 \%$ of participants who were excluded. The results may have been different if these people had been included in the analysis although we did not find significant sociodemographic differences between those included or excluded. Third, since the data were based on self-report, reporting bias may exist. Fourth, as most participants were under 70 years old, there may have been too few subjects with psychiatric disorders typical of older individuals for us to study them adequately. However, our study is focused on older and middle-aged people since several European studies on aging used representative samples of individuals aged 50 
and older (Börsch-Supan, et al., 2005; Steptoe, et al., 2012; Whelan, et al., 2013). Fifth, measures from The Composite International Diagnostic Interview (CIDI) were found to have a high false-positive rate in validation studies using clinical samples (Kurdyak \& Gnam, 2005). However, other researchers suggested that the CIDI was designed for community-based samples and concluded that it is potentially useful for monitoring general mental health although it is not a substitute for specific mental health measures (Mawani \& Gilmour, 2010). Finally, the follow-up period was short and results could vary with a longer follow-up. Future studies in different settings and countries are needed to replicate our findings on the longitudinal associations in affective disorders, loneliness and social networks.

\section{Conclusions}

The results of this study have highlighted the difficulties of socially isolated older adults in reconstructing or improving the characteristics of their social networks. Only $9.7 \%$ of moderately or most isolated older people managed to improve their relational situation two years later and, among the most isolated, none improved to better than moderately isolated; a situation that may be progressively aggravated by feelings of loneliness. Both objective social isolation and loneliness factors have been found to be robust risk factors for depression and anxiety independently, which acts as a warning not to underestimate the subjective aspects of social isolation. However, it seems that reducing the prevalence of social isolation, which affects more than $30 \%$ of older adults in Ireland, is a challenge at least as important as alleviating loneliness, due to the difficulty of improvement without social interventions and their effect on mental health of older adults.

\section{References}


Achenbaum, W.A., Bengtson, V.L., 1994. Re-engaging the disengagement theory of aging: on the history and assessment of theory development in gerontology. Gerontologist 34, 756-63.

Barry, M., Van Lente, E., Molcho, M., Morgan, K., Mcgee, H., Barry, C., et al., 2009. SLAN 2007: Survey of Lifestyle, Attitudes and Nutrition in Ireland Mental Health and Social Wellbeing Report. Dublin, Ireland.

[WWW Document]. URL https://www.ucd.ie/t4cms/slan_wellbeing_report.pdf

Beekman, A.T., Bremmer, M.A., Deeg, D.J., van Balkom, A.J., Smit, J.H., de Beurs, E. et al., 1998. Anxiety disorders in later life: a report from the Longitudinal Aging Study Amsterdam. Int. J. Geriatr. Psychiatry 13, 717-26.

Berkman, L.F., Syme, S.L., 1979. Social networks, host resistance, and mortality: a nine-year follow-up study of Alameda County residents. Am. J. Epidemiol. 109, 186-204.

Blazer, D.G., 2003. Depression in late life: Review and commentary. Journals Gerontol. Ser. A Biol. Sci. Med. Sci. 58, 249-65.

Börsch-Supan, A., Hank, K., Jürges, H., 2005. A new comprehensive and international view on ageing: introducing the 'Survey of Health, Ageing and Retirement in Europe'. Eur. J. Ageing. 2, 245-253.

Byers, A.L., Yaffe, K., Covinsky, K.E., Friedman, M.B., Bruce, M.L., 2010. High Occurrence of Mood and Anxiety Disorders Among Older Adults. Arch. Gen. Psychiatry 67, 489-496.

Cacioppo, J. T., Cacioppo, S. (2018). Loneliness in the Modern Age: An Evolutionary Theory of Loneliness (ETL). Adv. Exp. Soc. Psychol. 58, 127-197.

Cacioppo, J.T., Hawkley, L.C., Thisted, R., 2010. Perceived social isolation makes me sad: 5-year cross-lagged analyses of loneliness and depressive symptomatology in 
the Chicago Health, Aging, and Social Relations Study. Psychol. Aging 25, 453463.

Cohen-Mansfield, J., Hazan, H., Lerman, Y., Shalom, V., 2016. Correlates and predictors of loneliness in older-adults: a review of quantitative results informed by qualitative insights. Int. Psychogeriatr. 28, 557-76.

Cornwell, E.Y., Waite, L.J., 2009. Social disconnectedness, perceived isolation, and health among older adults. J. Health Soc. Behav. 50, 31-48.

Cotterell, N., Buffel, T., Phillipson, C., 2018. Preventing social isolation in older people. Maturitas 113, 80-84.

Cramer, V., Torgersen, S., Kringlen, E., 2005. Quality of life and anxiety disorders: a population study. J. Nerv. Ment. Dis. 193, 196-202.

Dahlberg, L., Andersson, L., Lennartsson, C., 2018. Long-term predictors of loneliness in old age: results of a 20-year national study. Aging Ment. Heal. 22, 190-196.

Dahlberg, L., Andersson, L., McKee, K.J., Lennartsson, C., 2014. Predictors of loneliness among older women and men in Sweden: A national longitudinal study. Aging Ment. Health. 7863, 1-9.

Djernes, J.K., 2006. Prevalence and predictors of depression in populations of elderly: A review. Acta Psychiatr. Scand. 113, 372-387.

Domènech-Abella, J., Lara, E., Rubio-Valera, M., Olaya, B., Moneta, M.V., RicoUribe, et al., 2017a. Loneliness and depression in the elderly: the role of social network. Soc. Psychiatry Psychiatr. Epidemiol, 52, 381-390.

Domènech-Abella, J., Mundó, J., Lara, E., Moneta, M.V., Haro, J.M., Olaya, B., 2017 b. The role of socio-economic status and neighborhood social capital on loneliness among older adults: evidence from the Sant Boi Aging Study. Soc. Psychiatry Psychiatr. Epidemiol. 52, 1237-1246. 
Domènech-Abella, J., Perales, J., Lara, E., Moneta, M.V., Izquierdo, A., Rico-Uribe, L.A., et al., 2017c. Sociodemographic Factors Associated With Changes in Successful Aging in Spain: A Follow-Up Study. J. Aging Health. 30, 1244-1262.

Harvey, B., \& Walsh, K., 2016). Loneliness and Ageing: Ireland, North and South. Dublin: Institute of Public Health in Ireland. [WWW Document]. URL https://www.publichealth.ie/document/iph-report/loneliness-and-ageing-irelandnorth-and-south

Hawkley, L.C., Louise, C., Cacioppo, J.T., 2010. Loneliness matters: a theoretical and empirical review of consequences ans mechanisms. Ann. Behav. Med. 40, 218227.

Houtjes, W., Van Meijel, B., Van De Ven, P.M., Deeg, D., Van Tilburg, T., Beekman, A., 2014. The impact of an unfavorable depression course on network size and loneliness in older people: A longitudinal study in the community. Int. J. Geriatr. Psychiatry 29, 1010-1017.

Jacobson, N.C., Newman, M.G., 2017. Anxiety and depression as bidirectional risk factors for one another: A meta-analysis of longitudinal studies. Psychol. Bull., $143,1155-1200$.

Jacobson, N. C., Lord, K. A., \& Newman, M. G., 2017b. Perceived emotional social support in bereaved spouses mediates the relationship between anxiety and depression. J. Affect. Disord., 211, 83-91.

Jacobson, N. C., \& Newman, M. G. 2016. Perceptions of close and group relationships mediate the relationship between anxiety and depression over a decade later. Depress. Anxiety, 33(1), 66-74.

Kessler, R.C., Andrews, G., Mroczek, D., Ustun, B., Wittchen, H.-U., 1998. The World Health Organization Composite International Diagnostic Interview short-form 
(CIDI-SF). Int. J. Methods Psychiatr. Res. 7, 171-185.

Kurdyak, P. A., \& Gnam, W. H., 2005. Small signal, big noise: performance of the CIDI depression module. Can. J. Psychiatry, 50, 851-856.

Lamers, F., van Oppen, P., Comijs, H.C., Smit, J.H., Spinhoven, P., van Balkom, et al., 2011. Comorbidity Patterns of Anxiety and Depressive Disorders in a Large Cohort Study. J. Clin. Psychiatry 72, 341-348.

Landis, J.R., Koch, G.G., 1977. The Measurement of Observer Agreement for Categorical Data. Biometrics 33, 159-174.

Levula, A., Wilson, A., Harré, M., 2016. The association between social network factors and mental health at different life stages. Qual. Life Res. 25, 1725-1733.

Mawani, F. N., \& Gilmour, H., 2010. Validation of self-rated mental health. Health Rep., 21, 61-75.

Moussavi, S., Chatterji, S., Verdes, E., Tandon, A., Patel, V., Ustun, B., 2007. Depression, chronic diseases, and decrements in health: results from the World Health Surveys. Lancet (London, England) 370, 851-858.

Nicholson, N.R., 2012. A Review of Social Isolation: An Important but Underassessed Condition in Older Adults. J. Prim. Prev. 33, 137-152.

Pantell, M., Rehkopf, D., Jutte, D., Syme, S.L., Balmes, J., Adler, N., 2013. Social Isolation: A Predictor of Mortality Comparable to Traditional Clinical Risk Factors. Am. J. Public Health 103, 2056-2062.

Porensky, E.K., Dew, M.A., Karp, J.F., Skidmore, E., Rollman, B.L., Shear, M.K., et al., 2009. The Burden of Late-Life Generalized Anxiety Disorder: Effects on Disability, Health-Related Quality of Life, and Healthcare Utilization. Am. J. Geriatr. Psychiatry 17, 473-482. 
Rico-Uribe, L.A., Caballero, F.F., Olaya, B., Tobiasz-Adamczyk, B., Koskinen, S., Leonardi, M., et al., 2016. Loneliness, Social Networks, and Health: A CrossSectional Study in Three Countries. PLoS One 11, e0145264.

Routasalo, P.E., Savikko, N., Tilvis, R.S., Strandberg, T.E., Pitkälä, K.H., 2006. Social contacts and their relationship to loneliness among aged people - a populationbased study. Gerontology 52, 181-187.

Rubin, D. B., 2004. Multiple imputation for nonresponse in surveys (Vol. 81). John Wiley \& Sons.

Russell, D., 1996. UCLA Loneliness scale (Version 3): reliability, validity, and factor structure. J Pers Assess. 66, 20-40.

Santini, Z.I., Fiori, K.L., Feeney, J., Tyrovolas, S., Haro, J.M., Koyanagi, A., 2016. Social relationships, loneliness, and mental health among older men and women in Ireland: A prospective community-based study. J. Affect. Disord. 204, 59-69.

Santini, Z.I., Koyanagi, A., Tyrovolas, S., Mason, C., Haro, J.M., 2015. The association between social relationships and depression: A systematic review. J. Affect. Disord. 175, 53-65.

Saris, I.M.J., Aghajani, M., van der Werff, S.J.A., van der Wee, N.J.A., Penninx, B.W.J.H., 2017. Social functioning in patients with depressive and anxiety disorders. Acta Psychiatr. Scand. 136, 352-361.

Starr, L.R., Hammen, C., Connolly, N.P., \& Brennan, P.A., 2014. Does relational dysfunction mediate the association between anxiety disorders and later depression? Testing an interpersonal model of comorbidity. Depress. Anxiety, 31, 77-86.

Stepler, R., 2016. Smaller share of women Ages 65 and Older are Living Alone: More are living with spouse or children. Pew Research Center, Washington, D.C. 
[WWW Document]. URL http://www.pewsocialtrends.org/2016/02/18/smallershare-of-women-ages-65-and-older-are-living-alone/

Steptoe, A., Breeze, E., Banks, J., \& Nazroo, J., 2012. Cohort profile: the English longitudinal study of ageing. Int. J. Epidemiol., 42, 1640-1648.

Steptoe, A., Shankar, A., Demakakos, P., Wardle, J., 2013. Social isolation, loneliness, and all-cause mortality in older men and women. Pnas. 110, 5797-5801

Thoits, P.A., 2011. Mechanisms linking social ties and support to physical and mental health. J. Health Soc. Behav. 52, 145-61.

Toepoel, V., 2013. Ageing, Leisure, and Social Connectedness: How could Leisure Help Reduce Social Isolation of Older People? Soc. Indic. Res. 113, 355-372.

Vink, D., Aartsen, M.J., Schoevers, R.A., 2008. Risk factors for anxiety and depression in the elderly: A review. J. Affect. Disord. 106, 29-44.

Wang, J., Mann, F., Lloyd-Evans, B., Ma, R., Johnson, S., 2018. Associations between loneliness and perceived social support and outcomes of mental health problems: a systematic review. BMC Psychiatry 18, 156.

Whelan, B.J., Savva, G.M., 2013. Design and Methodology of The Irish Longitudinal Study on Ageing. J. Am. Geriatr. Soc. 61, S265-S268. 
Table1. Characteristics of the study sample.

\begin{tabular}{|c|c|}
\hline (n) & $\begin{array}{l}\mathrm{n}(\%) \\
5066(100)\end{array}$ \\
\hline \multicolumn{2}{|l|}{ Sex } \\
\hline Female & $2775(51.8)$ \\
\hline \multicolumn{2}{|l|}{ Age cohorts } \\
\hline $50-59$ & $2133(43.2)$ \\
\hline $60-69$ & 1744 (33.1) \\
\hline $70-79$ & 961 (18.4) \\
\hline $80+$ & $228(5.3)$ \\
\hline \multicolumn{2}{|l|}{ Place of residence } \\
\hline Rural & 2775 (49.7) \\
\hline \multicolumn{2}{|l|}{ Employment } \\
\hline Employed & 1989 (39.0) \\
\hline Retired / disabled & $2021(38.1)$ \\
\hline Unemployed & $234(5.5)$ \\
\hline Homemaker & 731 815.7) \\
\hline Other & $91(1.7)$ \\
\hline \multicolumn{2}{|l|}{ Financial strain } \\
\hline Often & $830(19.0)$ \\
\hline Sometimes & $1818(37.1)$ \\
\hline Rarely & $1195(22.3)$ \\
\hline Never & $1134(22.7)$ \\
\hline \multicolumn{2}{|l|}{ Education } \\
\hline Primary & $1233(33.2)$ \\
\hline Secondary & 2120 (45.9) \\
\hline Tertiary & $1713(20.9)$ \\
\hline \multicolumn{2}{|l|}{ Heart diseases } \\
\hline 0 & $1848(36.2)$ \\
\hline $1-2$ & 2675 (52.9) \\
\hline $3-7$ & $543(10.9)$ \\
\hline \multicolumn{2}{|l|}{ Other diseases } \\
\hline 0 & $2628(52.2)$ \\
\hline $1-2$ & $2266(44.2)$ \\
\hline $3-7$ & $172(3.6)$ \\
\hline \multicolumn{2}{|l|}{ Affective disorders } \\
\hline Depression & $289(5.9)$ \\
\hline Anxiety & $142(2.8)$ \\
\hline \multicolumn{2}{|l|}{ Loneliness (0-10) } \\
\hline 0 & $1726(36.6)$ \\
\hline $1-2$ & 1488 (32.9) \\
\hline $3-4$ & 765 (16.9) \\
\hline $5-6$ & $412(9.4)$ \\
\hline $7-8$ & $120(2.9)$ \\
\hline $9-10$ & $60(1.3)$ \\
\hline \multicolumn{2}{|l|}{ Social Network Index } \\
\hline Most isolated & $356(7.1)$ \\
\hline Moderately isolated & $1327(26.2)$ \\
\hline Moderately integrated & 2079 (41.0) \\
\hline Most integrated & $1304(25.7)$ \\
\hline Widow(er) & 600 (11.9) \\
\hline
\end{tabular}


Table 2. Percentages of worsening, unchanged, and improving from wave 2 to wave 3.

\begin{tabular}{ccccccc}
\hline & \multicolumn{2}{c}{ Status in Wave 2 } & \multicolumn{3}{c}{ Change in Wave 3 } & Kappa w2-w3 \\
\hline Variable & Categories & $\mathrm{n}(\%)$ & Worsening & No change & Improving & \\
\hline \multirow{2}{*}{ Loneliness } & $0-4$ & $3979(86.4)$ & $22.6 \%$ & $53.70 \%$ & $23.70 \%$ & 0.31 \\
& $5-10$ & $592(13.6 \%)$ & $9.10 \%$ & $26.50 \%$ & $64.40 \%$ & \\
\multirow{2}{*}{ SNI } & Isolated & $1683(33.3)$ & $14.10 \%$ & $76.20 \%$ & $9.70 \%$ & \multirow{2}{*}{0.61} \\
& Integrated & $3383(66.7)$ & $23.60 \%$ & $70.60 \%$ & $5.80 \%$ & \\
\multirow{2}{*}{ MDD } & Presence & $289(5.9)$ & - & $77.20 \%$ & $22.80 \%$ & \multirow{2}{*}{0.21} \\
& Absence & $4777(94.1)$ & $3.90 \%$ & $96.10 \%$ & - & \\
\multirow{2}{*}{ GAD } & Presence & $142(2.8)$ & - & $74.90 \%$ & $25.10 \%$ & 0.25 \\
& Absence & $4924(97.2)$ & $2.10 \%$ & $97.90 \%$ & - &
\end{tabular}

Categories referred to as isolated include most and moderately isolated whereas those referred to as integrated include most and moderately integrated. Improvements in loneliness and SNI, according to the categories in Table 1, mean getting close to 0 or the "most integrated" category, respectively. In the case of MDD and GAD, improving means progressing from suffering to not suffering. In all cases, worsening means the opposite. Kappa agreement coefficient was also calculated with Table 1 categories. $\mathrm{SNI}=$ social network index. MDD=major depressive disorder in the past 12 months. GAD=generalized anxiety disorder in the past12 months. 
Table 3. Bivariate logistic regression models of the factors related to changes in depression, anxiety, loneliness and social network index (SNI).*

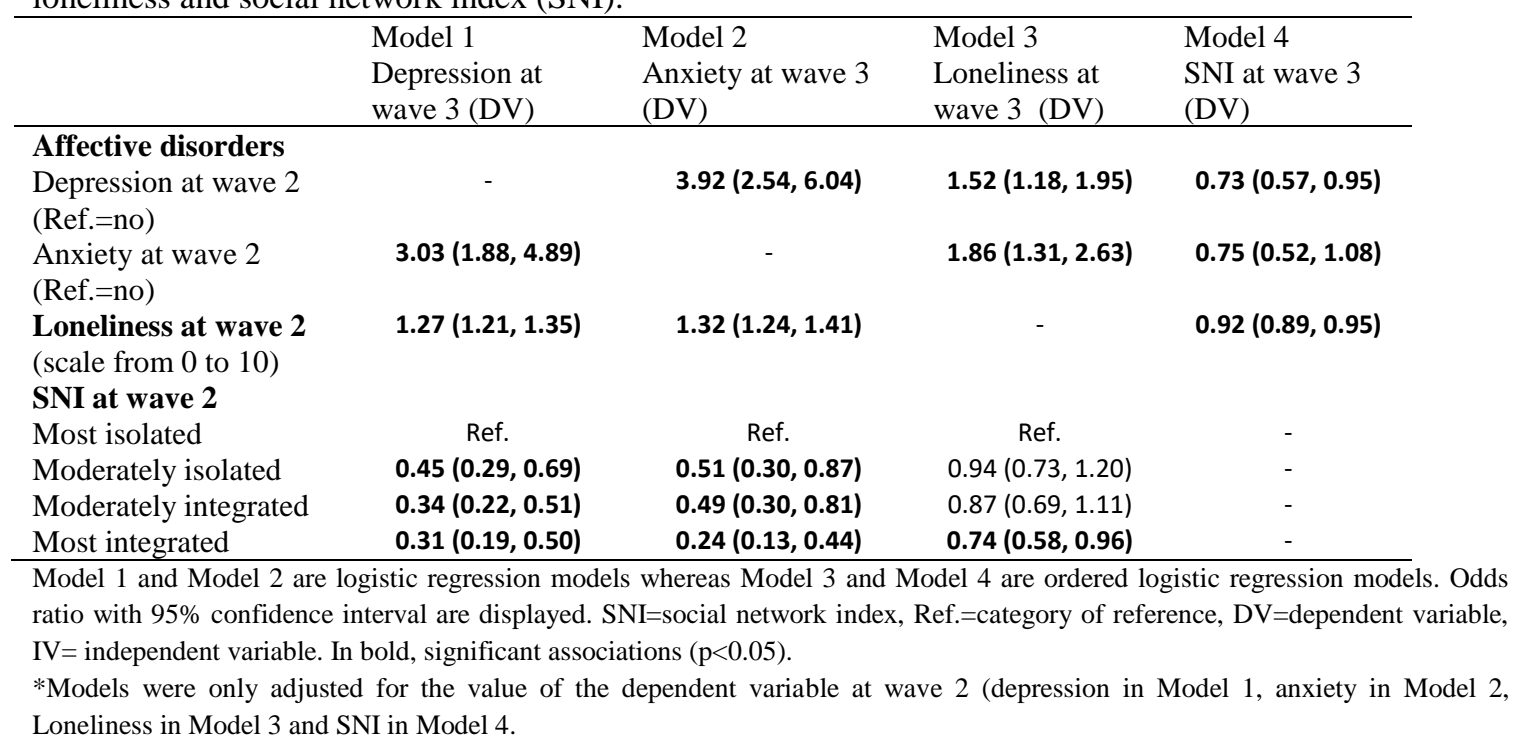


Table 4. Adjusted logistic regression models of the factors related to changes in depression, anxiety, loneliness and social network index (SNI).*

\begin{tabular}{|c|c|c|c|c|}
\hline & $\begin{array}{l}\text { Model } 1 \\
\text { Depression at } \\
\text { wave } 3 \text { (DV) }\end{array}$ & $\begin{array}{l}\text { Model } 2 \\
\text { Anxiety at wave } 3 \\
\text { (DV) }\end{array}$ & $\begin{array}{l}\text { Model } 3 \\
\text { Loneliness at } \\
\text { wave } 3 \text { (DV) } \\
\end{array}$ & $\begin{array}{l}\text { Model } 4 \\
\text { SNI at wave } 3 \\
\text { (DV) }\end{array}$ \\
\hline \multicolumn{5}{|l|}{ Affective disorders } \\
\hline $\begin{array}{l}\text { Depression at wave } 2 \\
\text { (Ref.=no) }\end{array}$ & - & $2.32(1.48,3.65)$ & $1.18(0.91,1.52)$ & $0.85(0.65,1.12)$ \\
\hline $\begin{array}{l}\text { Anxiety at wave } 2 \\
\text { (Ref.=no) }\end{array}$ & $2.03(1.24,3.33)$ & - & $1.60(1.10,2.34)$ & $0.93(0.63,1.37)$ \\
\hline $\begin{array}{l}\text { Loneliness at wave } 2 \\
\text { (scale from } 0 \text { to } 10 \text { ) }\end{array}$ & $1.22(1.15,1.30)$ & $1.24(1.16,1.33)$ & - & $0.93(0.90,0.96)$ \\
\hline \multicolumn{5}{|l|}{ SNI at wave 2} \\
\hline Most isolated & Ref. & Ref. & Ref. & - \\
\hline Moderately isolated & $0.58(0.37,0.92)$ & $0.69(0.40,1.20)$ & $1.06(0.83,1.35)$ & - \\
\hline Moderately integrated & $0.51(0.33,0.81)$ & $0.82(0.48,1.40)$ & $0.99(0.78,1.25)$ & - \\
\hline Most integrated & $0.57(0.34,0.96)$ & $0.48(0.25,0.95)$ & $0.85(0.66,1.10)$ & - \\
\hline
\end{tabular}


Figure 1. Flowchart of the study sample.

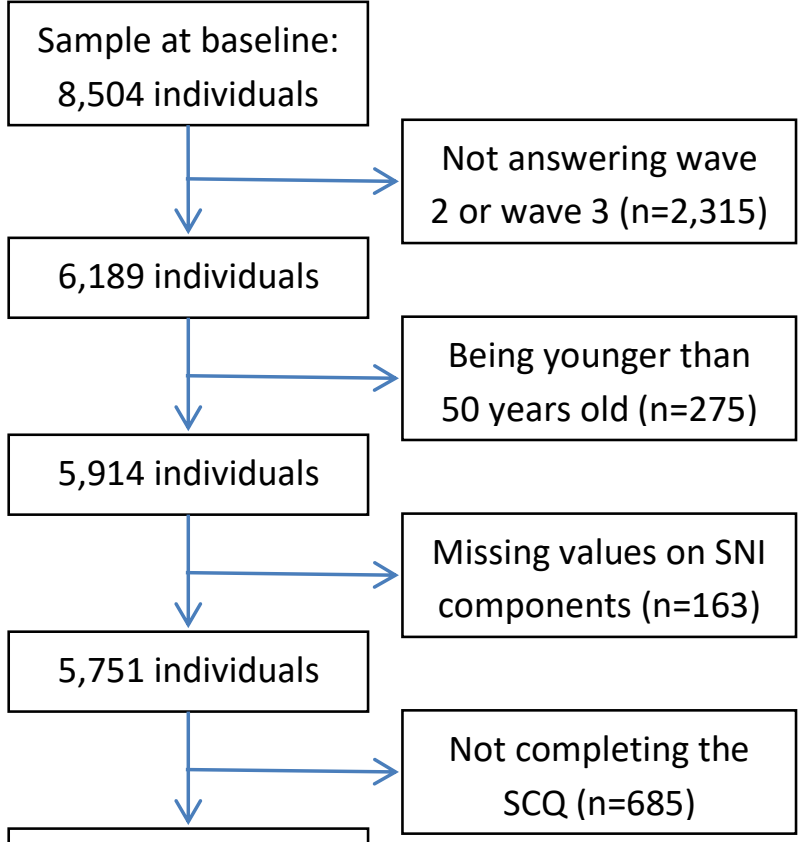

Study sample:

5.066 individuals 